\title{
Development of a Smartphone-Enabled Spirometer for Personalised Respiratory Health
}

\author{
Charalampos Michailidis ${ }^{+}$, Ioannis Smanis ${ }^{+}$, Kostas Stamatis, Christos Bergeles ${ }^{\star}$, Antonios Kouris
}

\begin{abstract}
Airway pathologies are among the most prevalent chronic diseases, and, despite their soaring strain on the healthcare system, are still responsible for millions of deaths worldwide. The lack of personalised respiratory health management systems prohibits individuals from monitoring their own condition, thus forcing them to rely on hospital visits. The presented spirometer aims to enable individuals to perform medical-grade respiratory tests using their smartphone.
\end{abstract}

Index Terms - Respiratory, health, mobile, mhealth.

\section{BREATHING: EXPENSIVE AND DEADLY}

Chronic Airway diseases, of which Chronic Asthma and Chronic Obstructive Pulmonary Disease (COPD) are the most pervasive representatives, affect more than 18.5 million [1], and 13 million [2] people in the United States alone, respectively, with a tendency to increase. Moreover, there is evidence of a staggering cost of 130 billion annually [3], [4].

Mobile health practices represent a paradigm shift in disease management, and empower individuals to monitor their own conditions, allowing in situ evidence-based medicine. It can be argued that chronic diseases such as diabetes, individually monitored through glucose meters, and hypertension, individually monitored through blood-pressure measurements, are now successfully controlled due to the existence of inexpensive and easy-to-use screening systems. This paper presents the design and calibration considerations for a smartphone-enabled spirometer, which we call Respi, aiming to empower patients to integrate accurate assessments of their pulmonary function into management of their disease. Respi aims to disrupt the information gap that physicians face when providing therapy, who do so in the absence of live data of the patient's lungs.

\section{Personalising Respiratory Health}

Chronic respiratory disease management requires both long term and short term action, mainly medication. Achieving steady state control is a continuous process and a possibly life threatening exacerbation can take place unexpectedly within hours. Physicians have entrusted patients to self report on symptoms and act accordingly, especially in case of an attack. The main symptoms a patient with respiratory disease faces are shortness of breath, tight chest feeling, wheezing

\footnotetext{
Respi, Inc., has been supported by SFEE (Hellenic Association of Pharmaceutical Companies) and DreamIt Health Baltimore.

Charalampos Michailidis and Ioannis Smanis are equally contributing co-first authors. Corresponding author: C. Bergeles c.bergeles@resp.io. All authors are affiliated with Respi, Inc., Baltimore, MD, USA.
}

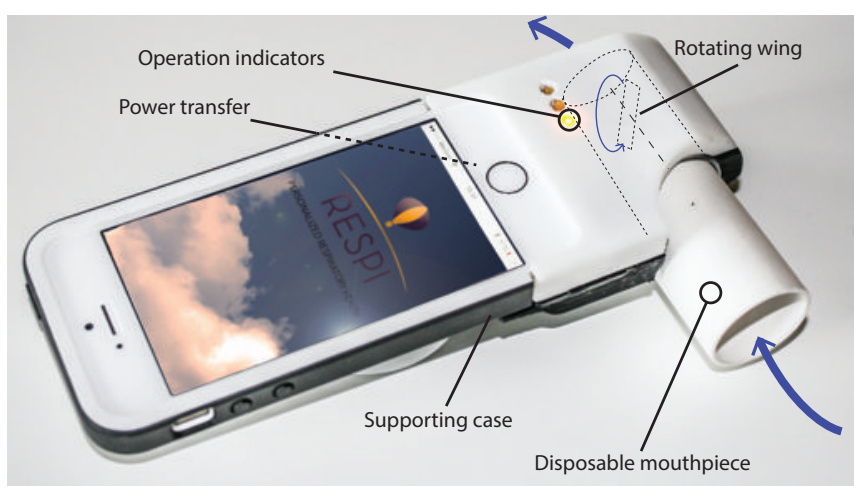

Fig. 1. Prototypical Respi spirometer connected to an iPhone ${ }^{\circledR} 5 \mathrm{~s}$.

and coughing and in cases of extreme emergency bluish facial colour, rapid pulse, sweating and decreased alertness.

Given that self-reported symptoms are used to base decisions on self-medication and hospital admissions and that physicians introduce those reports to base patients' therapy, it has been a long effort to quantify them. To this extent, several non medically certified devices have been administered to patients, so that they can base decisions and track records.

The initial quantification effort has been the PEF meter, a mechanically operated device, which can record the maximum flow that a patient can exhale. Lately, electronic versions have appeared with the added benefits of accuracy and record tracking. Several reports [5], [6] that these devices fail to provide concordant measurements and no ageing studies exist to certify robustness of operation with usage. Philips-Respironics Assess Peak Flow Meter that is operated pneumatically and the smartphone enabled MySpiroo (under development) are two indicative devices.

Several devices have been proposed to assess the complete respiratory condition of a patient, which perform a proper standardised test or a limited version of it. Numerous small sized, medically certified spirometers are available in the market and are being reimbursed by several healthcare systems. Though physicians advice patients to use them often and pertain health decisions based on live measurement, home-based spirometers are usually not transportable. These spirometers also have little sanity control against bad measurements (e.g. artefacts made from coughing while measuring), do not record patient context (e.g. time and type of last medication), and fail to provide a deep pulmonary record of the patient for use by the pulmonary specialist. The PC-based MIR Minispir, the standalone MIR Spirodoc 


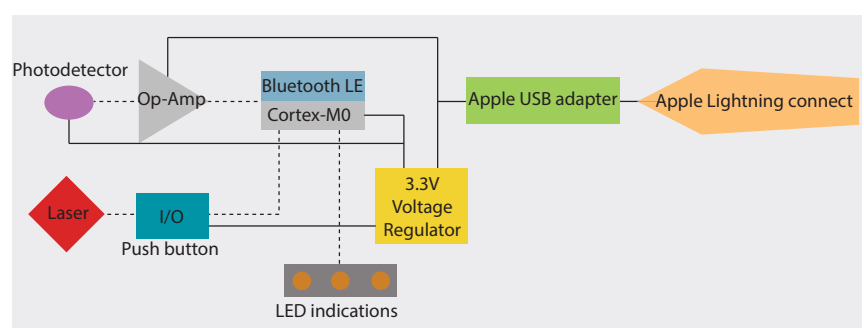

Fig. 2. Illustration of the electronics connections.

and the smartphone enabled Wello (announced production) are two indicative devices in the space.

Other proposals are devices with atypical measurements, usually in collaboration with the user's smartphone, such as AirSonea [7] which makes a sound record of the user's airway track and detects wheezing via a cloud-based software, and SpiroSmart [8], which measures expiratory parameters using sounds recorded from the microphone. Inherently, though, microphone-based measurements are sensitive to environmental noise.

Propeller Health are aggregating a pattern based on geotagging the user's intake of rescue medication contained in a Metered-Dose Inhaler. Whereas there are indications that the measurements aggregated by these devices are complimentary to the usual physician input, there is little document evidence of their significance.

The spirometer proposed in this paper is innovative in both the form factor and on the user proposition. It is the first standalone spirometer to fully utilise the processing power, display and battery of the smartphone, and, unlike all devices, it's direct connectivity to the smartphone will offer the option for actionable advice to patients, through user conscious UI and personalised analytics, and the ability to create a rich, context-aware, pulmonary profile for use by physicians. In the following the design and calibration considerations are presented.

\section{Design And Calibration Considerations}

\section{A. Spirometry Basics}

Spirometry is the standardised clinical test that measures how an individual's lungs function. Specifically, it measures the flow over time that can be inhaled and exhaled, and represents it through a Flow-Volume graph from which one can export the following key values [9] (after maximal inspiration): Forced Vital Capacity (FVC) [L], i.e. the maximal volume of air exhaled with maximally forced effort; Forced Exhaled Volume (FEVt) [L], i.e. the maximal volume of air exhaled in the first $t$ seconds of a forced expiration; Peak expiratory flow (PEF) $[\mathrm{L}]$, i.e. the maximal flow achieved during the maximally forced expiration.

\section{B. Spirometer Design Considerations}

Stringent design considerations have being laid forth and enforced during the design of Respi. First, the spirometer should be powered by the smartphone, but should rely

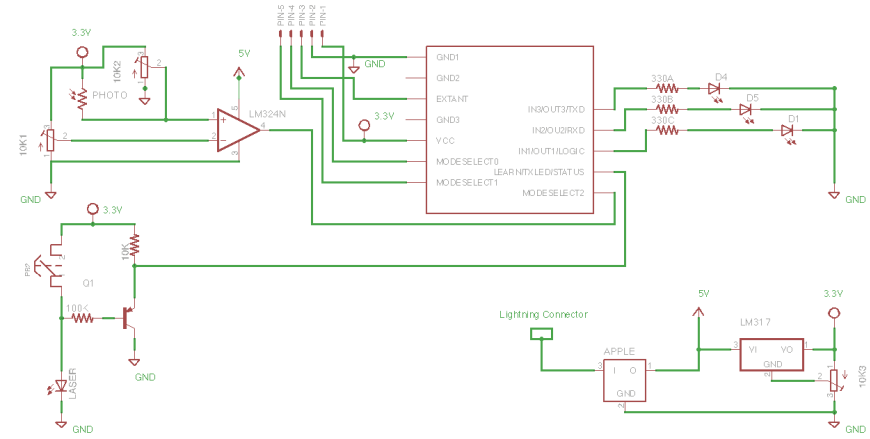

(a)

Fig. 3. Detailed schematic of the electronic components.

on existing wireless communication mechanisms in order for it to be deployable across the smartphone spectrum without adaptations. An ubiquitous communication means is Bluetooth 4.0.

Second, the spirometer should have disposable mouthpieces in order to achieve a low cost and increase its portability. Hence, the disposable mouthpieces from Medical International Research (MIR), Inc. were selected (see Fig. 3(a)). Selecting existing mouthpieces has the added advantage that our device is building on top of existing approved medical components, which reduces the regulatory burden. The selected mouthpiece includes a lightweight wing, indicated in Fig. 3(a). The speed of rotation of the lightweight wing depends on the air flow through the disposable turbine. Even though the fabrication method of the disposable turbines is patented [10], measuring the revolutions of a rotating body is a common mechanism in spirometry. Prior to patenting our proprietary mechanism, we are using a light-based approach.

Third, preliminary fluid dynamics simulations indicated a revolution of the wing at rates on the order of 10,000 RPM. This corresponds to revolutions on the order of $160 \mathrm{~Hz}$, which, based on Nyquist criterion, requires a sampling rate at the minimum of $\mathrm{f}_{\mathrm{N}}=320 \mathrm{~Hz}$. Hence, electronic components and signal processing methods should be selected appropriately. As Fig. 2 shows, the main part of the electronics is the Nordic nRF51822 SoC which is a powerful 32bit ARM Cortex M0 microcontroller running $16 \mathrm{MHz}$. Analog-to-digital conversion occurs at $\mathrm{f}_{\mathrm{S}}=658 \mathrm{KHz}$ and 8 bit per sample. The sampling rate is larger than the Nyquist frequency $\left(f_{N}\right)$, and ensures the reliability of the converted analog signal from the quick response photo-resistor.

The particular photo-resistor has a peak-to-peak response time of less than $10 \mathrm{~ms}$. By implementing a voltage comparator with an operational amplifier (Op-Amp), our device takes advantage of an adjustable resistor (trimmer) to reduce the refresh rate of the photo-resistor. The trimmer is connected to the negative pin of the Op-Amp playing the role of the voltage reference in order to decrease the response time of the photo-resistor to approximately $2 \mathrm{~ms}$, again, satisfying the Nyguist criterion.

Finally, the Cortex-M0 calculates the revolution rates 
TABLE I

OPERATING VOLTAGE \% VS FLOW RATE.

\begin{tabular}{|c|c|c|c|c|c|c|c|c|c|}
\hline Operating point & $10 \%$ & $20 \%$ & $30 \%$ & $40 \%$ & $50 \%$ & $60 \%$ & $70 \%$ & $80 \%$ & $90 \%$ \\
\hline Average flow rate $[\mathrm{L} / \mathrm{s}]$ & 2.21 & 4.71 & 6.34 & 7.41 & 8.06 & 8.96 & 9.54 & 10.34 & 11.54 \\
\hline Std flow rate $[\mathrm{L} / \mathrm{s}]$ & 0.066 & 0.040 & 0.025 & 0.028 & 0.020 & 0.030 & 0.023 & 0.040 & 0.024 \\
\hline$\%$ variation & 2.9 & 0.8 & 0.4 & 0.4 & 0.2 & 0.3 & 0.25 & 0.4 & 0.2 \\
\hline
\end{tabular}

TABLE II

FLOW RATE VS RPM FOR PROTOTYPE.

\begin{tabular}{|r|r|r|r|r|r|r|r|r|r|}
\hline Average flow rate [L/s] & 2.21 & 4.71 & 6.34 & 7.41 & 8.06 & 8.96 & 9.54 & 10.34 & 11.54 \\
\hline Average RPM & 5162 & 10422 & 14059 & 16560 & 18016 & 19936 & 21173 & 22772 & 25531 \\
Std RPM & 42 & 151 & 341 & 447 & 473 & 494 & 584 & 453 & 330 \\
\% variation & 0.8 & 1.4 & 2.4 & 2.7 & 2.6 & 2.5 & 2.7 & 1.9 & 1.3 \\
\hline
\end{tabular}

of the propeller, which is sent to the smartphone as a 4 byte value via bluetooth 4.0 . Bluetooth 4.0 , operating at $115.2 \mathrm{kbps}$, achieves a $3.6 \mathrm{kHz}$ transfer rate for the 4 bytes message. Hence, our electronics guarantee that there is no bottleneck from analog signal sampling stage to the smartphone packet reception.

Currently, the smartphone used is an iPhone $\AA$ s (see Fig. 3(a)), and power is transferred through a customised Apple Lightning connector. At this stage, our iOS application wirelessly transfers the data to a connected computer for post-processing, and there is no support for medical data encryption, which is under development. In full production we are estimating a price tag of $\$ 180$.

A detailed schematic of the electronics is given in Fig. 3.

\section{Spirometer Calibration}

Our calibration approach is designed to be against a standard-market spirometer, keeping FDA510(K) regulations in mind. Hence, ground truth is provided based on the values obtained from the MiniSpir Light of MIR. Airflow is provided by a servo controlled turbine, as has already been evaluated in the literature [11] (see Fig. 4). For different operating voltages of the servo controlled turbine, the airflow is calculated through MiniSpir in litres per second $(\mathrm{L} / \mathrm{s})$. Accuracy and repeatability are evaluated by performing sets of 10 measurements for each operating voltage, and repeating these experiments 10 times after dismantling and reassembling the calibration setup. Table I demonstrates that the process is repeatable and with high accuracy.

Quality assurance [12] in spirometer calibration requires that the spirometers are calibrated against flow rates starting from $2 \mathrm{~L} / \mathrm{s}$ and up to $8 \mathrm{~L} / \mathrm{s}$. Moreover, spirometry calibration checks require a repeatability on the order of $3.5 \%$ [12]. The operating-voltage to flow-rate mapping, extracted from through linear regression from the values of Table I, satisfies these criteria.

After extracting the operating-voltage to flow-rate mapping, the RPM to flow-rate map for our spirometer is identified. This achieved by substituting the market spirometer MiniSpir, which was use in the previous step, with our prototype. The calibration setup guarantees that the disposable mouthpiece remains fixed within the setup, and the only

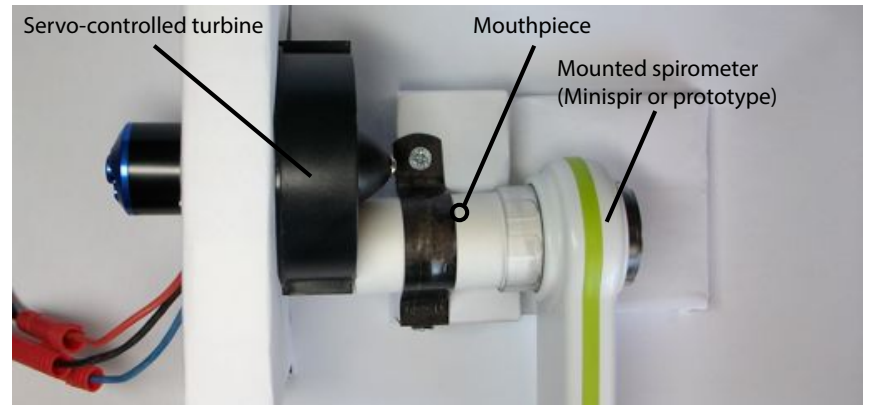

Fig. 4. Calibration setup.

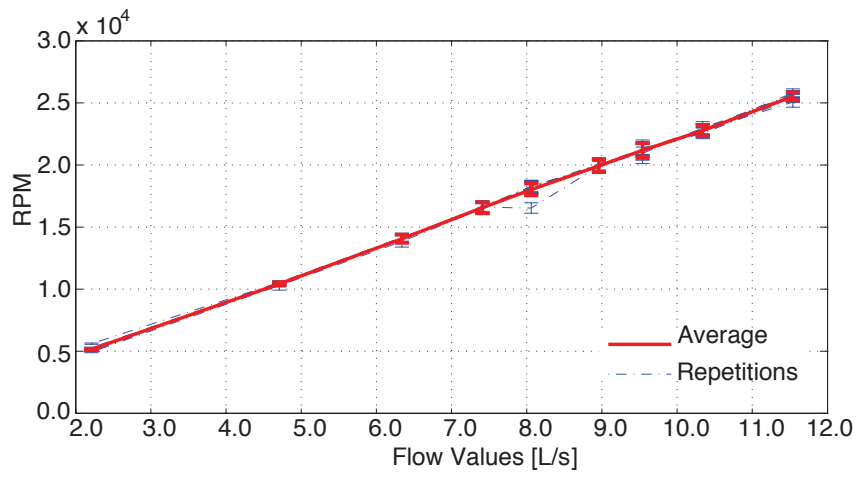

Fig. 5. Flow rate to RPM graph.

change is whether the market spirometer or our prototype is attached to it. Hence, no errors are expected to be introduced.

For the different flow rates generated by the turbine, the RPM measured by our spirometer is calculated. When steady-state is reached, this RPM value is associated with a particular flow rate. Fig. 5 depicts the RPM to flow rate relationship for our device. Due to the lack of inertia of the rotating wing in the spirometer, this linear relationship is expected. As before, repeatability is evaluated by repeating this process 10 times (see Table II). Linear regression based on this data enables the calculation of flow rates from the RPM data, hence, completing the calibration of our device. 


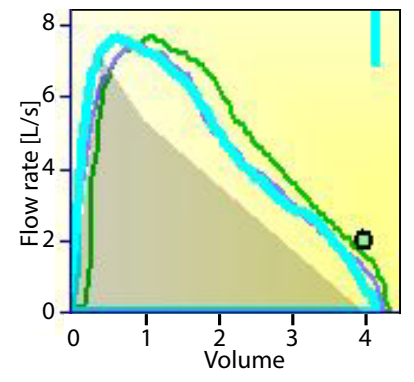

(a)

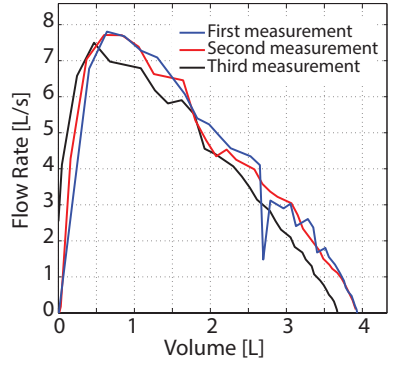

(b)
Fig. 6. (a) Spirometry using MiniSpir, and (b) Spirometry using our prototype.

TABLE III

COMPARISON OF RESPIRATORY PARAMETERS.

\begin{tabular}{|r|r|r|r|}
\hline Parameter & MiniSpir & Prototype & Error \% \\
\hline PEF [L/s] & 7.72 & 7.81 & $1.11 \%$ \\
FEV1 [L] & 4.15 & 3.71 & $10.5 \%$ \\
FEV6 [L] & 4.34 & 3.92 & $9.6 \%$ \\
FVC [L] & 4.34 & 3.92 & $9.6 \%$ \\
FEV1\% & 86.3 & 94.71 & $0.9 \%$ \\
\hline
\end{tabular}

\section{Experiments}

A young female volunteer performed self-spirometries using the commercial spirometer MiniSpir and our device (see Fig. 6). Table III demonstrates that the error between the extracted physiological parameters is maximally $10 \%$, dropping to $1 \%$ when PEF is considered. These errors are very encouraging, especially in light of intra-spirometry variability due to the straining of a subjects respiratory system being up to $7 \%$ [13]. Hence, the smartphone-enabled spirometer, created from low-cost off-the-self components, has the potential to become an important device in personalising respiratory care.

\section{Conclusions And Discussion}

This paper presented a novel smartphone-enabled spirometer that aims to empower users to take control of their respiratory health, by providing them with an easy to use and cost-effective platform for self monitoring. Even though at early stages of development, this paper demonstrates that the proposed mechatronics design is viable. Based on off-the-self components and existing medically approved mouthpieces, it can easily fit in the market.

Additional aspects of this project include the creation of deep pulmonary profiles for individuals, something which is impossible with existing devices the lack of personalised data. This information will allow monitoring of an individual's respiratory health, and communication with the clinician when the necessity arises.

\section{REFERENCES}

[1] D. L. Hoyert and J. Xu, "Deaths: preliminary data for 2011," National vital statistics, vol. 61, no. 6, pp. 1-51, 2012.

[2] Centers for Disease Control and Prevention. (2014) Facts about asthma. [Online]. Available: http://www.cdc.gov/nchs/fastats/asthma. htm
[3] American Lung Association. (2014) Chronic obstructive pulmonary disease (COPD) fact sheet. [Online]. Available: http://www.lung.org/ lung-disease/copd/resources/facts-figures/COPD-Fact-Sheet.html

[4] The New York Times. (2014, October) The soaring cost of a simple breath. [Online]. Available: http://www.nytimes.com/2013/10/13/us/ the-soaring-cost-of-a-simple-breath.html?pagewanted=all\&_r=0

[5] G. N. Takara, G. Ruas, B. V. Pessoa, L. K. Jamami, V. A. Di Lorenzo, and M. Jamami, "Comparison of five portable peak flow meters," Clinics (Sao Paolo), vol. 65, no. 5, pp. 469-474, 2010.

[6] B. O. Adeniyi, V. C. Moore, G. E. Erharbor, and S. Burge, "Differences in serial lung function recorded on four data-logging meters," $J$. Asthma, vol. 50, no. 9, pp. 965-967, 2013.

[7] Airsonea. (2014) Air Sonea. [Online]. Available: www.airsonea.com

[8] E. C. Larson, G. Mayank, G. Boriello, S. Heltshe, M. Rosenfeld, and S. N. Patel, "Spirosmart: using a microphone to measure lung function on a mobile phone," ACM Int. Conf. Ubiquitous Computing, pp. 280289, 2012.

[9] M. R. Miller, J. A. T. S. Hankinson, V. Brusasco, F. Burgos, R. Casaburi, A. Coates, R. Crapo, P. Enright, C. P. Van der Griten, and P. Gustafsson, "Standardisation of spirometry," European Respiratory Journal, vol. 26, no. 2, pp. 319-338, 2005.

[10] P. B. Sacco, "Disposable spirometer with plastic injection moulded turbine," US Pattent 7618235, 2009.

[11] G. Saumon, A. Loiseau, and E. Delavault, "Servo-controlled air pump for calibration of respiratory measurement systems," J. Biomedical Engineering, vol. 7, pp. 132-136, 1985.

[12] College of Physicians and Surgeons of British Columbia, "Diagnostic accreditation program in spirometry: quality control plan," pp. 1-20, 2012.

[13] A. Kainu, A. Lindqvist, S. Sarna, and A. Sovijarvi, "Intra-session repeatability of FET and FEV6 in the general population," Clinical Physiology and Functional Imaging, vol. 28, no. 3, pp. 196-201, 2008. 